MAGNETOHYDRODYNAMIC TURBULENCE IN THE SOLAR WIND

by

William H. Matthaeus and Melvyn L. Goldstein

Laboratory for Extraterrestrial Physics

Code 692, NASA/Goddard Space Flight Center, Greenbelt, MD 20771

\begin{abstract}
Recent work in describing the solar wind as an MHD turbulent fluid has shown that the magnetic fluctuations are adequately described as time stationary and to some extent as spatially homogeneous. Spectra of the three rugged invariants of incompressible MHD are the principal quantities used to characterize the velocity and magnetic field fluctuations. Unresolved issues concerning the existence of actively developing turbulence are discussed.
\end{abstract}

\title{
INTRODUCTION
}

To describe a fluid system as turbulent is to say that the dynamical fluid variables exhibit complex and essentially non-reproducible behavior as a function of time. This is generally due to the presence of nonlinearities in the fluid equations which strongly couple a large number of degrees of freedom. Turbulent systems are usually very far from equilibrium states for which detailed analytically tractable theories might exist.

By all appearances, the solar wind plasma flow and the interplanetary magnetic field carried along with it are such a turbulent system. In the zero momentum frame, the magnetic and velocity field fluctuations are energetically comparable to the mean magnetic field over length scales of order $1 \mathrm{AU}$ and display the type of complicated behavior expected of turbulence.

The prospect that plasma turbulence techniques may be applied and developed in the context of solar wind studies is attractive. Although the interplanetary medium cannot be controlled in the ways a laboratory plasma might be, it does persist in time in what might be thought to be a statistically steady state. More importantly, interplanetary exploration has provided us with a vast data base of magnetic field and plasma properties along spacecraft trajectories. The availability of these data, reduced to a form compatible with the language of turbulence theory, now provides the opportunity to evaulate existing theories and may provide guidelines for the development of new approaches. In this paper, we summarize some of our recent work [Matthaeus and Goldstein, 1982a,b] which begins to address the questions: to what extent does the interplanetary medium resemble a turbulent MHD medium and what can one learn about the validity of turbulence theory from experimental space physics.

The theoretician studying turbulence properties of the interplanetary medium must deal primarily with appropriately defined ensemble averaged properties of the interplanetary fields [Batchelor, 1970]. The connection between statistical theories of turbulence and experimental reality can be made only when it is possible to show that the theoretician's abstract notion 
of an ensemble has a plausible correspondence to some experimentally implemented averaging procedure. The connection usually sought is that time averages of the products of the turbulent fields at fixed points in space are equivalent, in practice, to ensemble averaging. When this is so, the fluctuations are 'ergodic'. If time averages are also insensitive to the origin in time, the fluctuations are statistically stationary. As a first step in deciding whether it is even appropriate to describe interplanetary fluctuations using turbulence theory, we establish that the interplanetary magnetic field often behaves as a stationary and ergodic random function of time.

\section{TIME STATIONARITY}

Consider a component of the magnetic field, $B(t)$, at a fixed point in space. Frequency spectra and two-time correlation functions indicate that $B(t)$ consists of a continuum of frequency components superposed on coherent signals with periods of the solar rotation period and its first few harmonics. The signals commensurate with the solar rotation are indicative of sector structure, stream structure and other nearly periodic phenomena relating to the origin of the solar wind and its possible continued forcing by high speed streams.

Stationarity of the average field, $\langle B(t)\rangle=a$, and second order moments such as the correlation function $R(\tau)=\langle B(t) B(t+\tau)\rangle$ is just the property that the ensemble average denoted by the brackets $\langle\ldots\rangle$ does not depend on $t$. To determine the extent to which this property is reflected in the data, we consider sequences of time averages of the field covariances of duration $T$. According to the ergodic theorem [Panchev, 1971] for stationary random processes, the mean values of these averages converge to the ensemble means in a calculable fashion. We have shown [Matthaeus and Goldstein, 1982b] that the expected variance of the time averages of $B(t)$, say $[B(t)]_{T}$, is, for large $T$

$$
\Delta^{2}[B]_{T}=\left\langle\left([B]_{T}-a\right)^{2}\right\rangle=2 \sigma^{2} T / T_{c}+4 \sigma_{0}^{2} \sin ^{2}\left(\omega_{0} T / 2\right) /\left(\omega_{0} T\right)^{2}
$$

when coherent power at frequency $\omega_{0}$ is properly taken into account. In (1), $\sigma^{2}$ is the incoherent power, $\sigma^{2}$ the coherent power and $T_{c}$ is the correlation time of $B(t)$. The right hand side depends only on bulk statistical properties of $B$. However, the left hand side can also be estimated from a large dataset by carrying out many time averages of duration $T$ and averaging the results. Comparing the asymptotic ensemble prediction in (1) with the averages from the data provides a test of the stationarity hypothesis.

The longest dataset we have analyzed in this manner utilized the $(X, Y, Z)$ $=$ (radial, tangential, normal) components of the interplanetary magnetic fleld taken from a 621 day IMP dataset in the National Data Center. The results show that the ergodic theorem is an excellent predictor of the behavior of the averages. The apparent convergence of the variance of estimates of $\langle B\rangle$ indicates that both first and second order moments are stationary since the mathematics leading to (1) requires that the two-time correlation $R(\tau)$ be independent of $t$. For this result to be useful, we also need to be able to obtain good estimates of second order moments from the same finite amount of data. This requirement is equivalent to using time averaging to obtain convergence of certain fourth-order moments of $B(t)$ (namely, the variance of estimates of the variance of $B$, each of which is a time average). This has al so been established for the IMP dataset.

The stationarity test has also been applied to a number of other magnetic field datasets using ISEE and Voyager data at heliocentric distances of $1-10$ 
AU. The length of the data records ranged from about 10 to 100 days. The degree of convergence of large $T$ time averages to predictions such as (1) was used as a figure of merit. These analyses indicate that 'good' convergence is obtained for datasets containing many ( $>10)$ correlation times, provided that significant organized structures such as isolated shocks and sector crossings are not undersampled. For example, a dataset spanning two or three sectors cannot be expected to give statistical estimates which are as well behaved as those obtained from a smaller subset of the data which includes no sector crossing.

These results suggest that the interplanetary magnetic field can be meaningfully viewed as a 'weakly' stationary random function. Strict stationarity requires that all moments, not just means and second order correlation functions, be independent of time.

It should be noted that the stationarity test described above is far from a mathematically rigorous procedure. One difficulty is that estimates of the left hand side of (1) utilize an average over a finite number of realizations of duration $T$ rather than an average over the entire ensemble. It was also necessary to estimate the values of the ensemble parameters $a, \sigma_{0}^{2}, \sigma^{2}$ and $T_{c}$ from the same finite span of data. The values of $T$ used were restricted to be less than one-fifth the total data record to minimize the effects of those approximations. We believe that this type of restriction is essential to prevent spurious results, though we have been unable to show this analytically.

\section{SPATIAL HOMOGENEITY}

Spatial homogeneity is another possible symmetry of turbulent fluctuations [Batchelor, 1970] and is particularly important if wavenumber spectral analysis is to be performed. Much of the existing turbulence theory deals with this case. Moreover, the dynamical processes which characterize homogeneous turbulence may play an important role in turbulence which is, at the largest scales, inhomogeneous. This statement is most easily justified in the very large Reynolds number case, in which turbulent fluctuations are found at all spatial scales ranging from energy containing scales down to a dissipation scale. If the length scales in this range are well separated from the lengths characterizing the scale of the entire system, homogeneity is a reasonable expectation.

In the most general case, time stationarity and spatial homogeneity are separate issues. However, the solar wind is a super-Alfvenic flow which allows the approximate identification,

$$
\langle B(\underline{x}, t) B(\underline{x}+\underline{r}, t)\rangle=\langle B(\underline{x}, t) B(\underline{x}, t+\tau)\rangle
$$

where $\underline{r}=-v_{\text {SW }} \underline{R}, v_{\text {sw }}$ is mean solar wind speed and $\underline{R}$ is the radial unit vector in heliocentric coordinates. This 'frozen-in flow' property is valid for phenomena occuring on a MHD time scale. If the time-stationary property holds at all points in space, (2) implies that $B(x, t)$ is also spatially homogeneous. Equation (2) cannot be valid for spatial separations $r$ which approach the scale over which the bulk heliospheric quantities vary, which can be plausibly taken to be the local heliocentric radial coordinate, $R$. In view of the results of the previous section, we have concluded that interplanetary MHD fluctuations at frequency $\omega$ correspond to spatially homogeneous structures provided that their scale $v_{s w} / \omega$ is much less than $R$. It seems reasonable from a theoretical point of view to assume that interplanetary fluctuations are both stationary and locally homogeneous with macroscopic parameters varying 
slowly, over scales of order $R$. This limited connection between stationarity and homogeneity can only be useful when the correlation length $L$ order that the spatial dependence of the fluctuations can be well sampled without encountering effects due to the scale size of the heliosphere.

\section{RUGGED INVARIANTS OF MHD IN THE SOLAR WIND}

Theoretical descriptions of fully developed MHD turbulence often dissuss the behavior of the 'rugged' invariants of the MHD equations. We have developed techniques for determining the rugged invariants of three dimensional MHD and their reduced wavenumber spectra from single spacecraft data [Matthaeus and Goldstein, 1982a]. The three known rugged invariants are the energy density, $E=\left\langle B^{2}+v^{2}\right\rangle$, the cross helicity, $H_{C}=\langle\underline{v} \cdot \underline{B}\rangle$, and the magnetic helicity, $H_{m}=\langle A \cdot B\rangle$. The energy density is the sum of magnetic and kinetic energy densities, with the magnetic field $B$ written in Alfven speed units. The magnetic helicity is the mean projection of the magnetic vector potential, $A$, on the magnetic field $B$. In the above definitions, $\underline{B}$ is taken to be the fluctuating field and $A$ is assumed to be in the coulomb gauge. These three quantities are invariants of ideal MHD and they are "rugged" because their invariance obtains for finite dimensional Galerkin-type representations of MHD, as well as the usual continuum model. In all cases tested so far [see for example, Fyfe and Montgomery, 1976] rugged invariants have been shown to be the isolating constants of motion necessary to describe the unphysical, but theoretically suggestive finite dimensional statistical mechanics models of turbulent ideal MHD and Navier stokes flows. In physically realistic turbulence, which always admits dissipation at the smallest spatial scales, rugged invariants are no longer constants of the motion. However their values are not changed by the action of nonlinearities in the equations of motion. Since nonlinear couplings alone induce transfer of excitations from one length scale to another, the set of rugged invariants imposes direct restrictions on how turbulent energy transfer occurs. For this reason much of turbulence theory is couched in the vocabulary of rugged invariants and their wavenumber spectra.

Here we shall not reproduce detailed analyses of data, but merely summarize the type of spectra which have most frequently been seen.

The energy spectra analyzed between 1 and 10 A.U. show power law wavenumber dependences of $k^{-a}$ with $a=-1.55$ to -1.7 . The power law region extends from scales near the correlation length, which is usually between $10^{12}$ and $10^{13} \mathrm{~cm}$, down to scales at least as small as our usual cutoff, about $10^{8}$ $\mathrm{cm}$. We have not yet analyzed data at high enough frequencies to resolve any sort of dissipation range where the spectrum is expected to steepen considerably. The power law is usually very near the Kolmogorov exponent of $-5 / 3$. The appearance of this type of power law dependence is strongly suggestive that a turbulent inertial range is being observed, even though the solar wind is probably quite anisotropic [Belcher and Davis, 1971] and the Kolmogorov spectral prediction presumes isotropy. Others have sometimes reported different power law dependences [Sari and Ness, 1969; Denskat, these proceedings], particularly for observations inside of $1 \mathrm{AU}$ where homogeneity is less likely a good approximation. It is also possible that at these smaller heliocentric distances the turbulence may not yet have had time to become fully developed.

Typical solar wind magnetic helicity spectra alternate in sign throughout the power law range and usually at lower wavenumbers as well. Because the magnetic helicity spectrum is a measure of the topological handedness of the 
fluctuations near a given wavenumber, the observed spectra indicate that the inertial range consists of both left and right handed magnetic structures. The net magnetic helicity for a "normal" solar wind period is that which is due to the largest scales sampled and the lengths characterizing the helicity scale are larger than the correlation length which is an estimate of the energy containing scale. This is similar to what one might expect in a steady state inverse cascade [Frisch et al., 1975] or freely decaying selective decay situation [Matthaeus and Montgomery, 1980]: MHD turbulence is thought to transfer magnetic helicity preferentially to the largest allowed scales. Near interplanetary shocks [Russell, these proceedings] and the terrestrial [Hoppe et al.. 1981] and Jovian [Smith et al., 1983] bow shocks the presence of circularly polarized waves is signaled by a distinct bias in the helicity spectra.

The cross helicity spectrum is a measure of the correlation between the velocity and magnetic fluctuations as a function of wavenumber. Similar quantities have been previously used [for example, see Coleman, 1967 and Belcher and Davis, 1971] to detect "Alfvenic fluctuations". In highly Alfvenic periods, the inertial range cross helicity is generally single-signed and attains a large fraction of its largest allowed values. However, it is not unusual to see the opposite sign of cross helicity at scales at or larger than the correlation length. In a wave interpretation, these wavenumber intervals correspond to inward-propagation, while the inertial range has a cross helicity indicating outward propagation. In at least one other interval, the cross helicity has been seen to be of mixed sign throughout the inertial range but with a fixed sign corresponding to outward propagation at the largest scales sampled.

\section{DISCUSSION}

We have attempted the beginnings of a systematic description of interplanetary fluctuations in a vocabulary appropriate to MHD turbulence theory despite serious questions which can be raised regarding the applicability of the model [see the review by D. Montgomery in these proceedings]. Even within the context of this model, there appear to be three distinct dynamical scenarios which might describe the observations.

The first of these is that, for one reason of another, the nonlinear couplings between the fluctuations are short circuited so that small amplitude wave theory encompasses the important physics. In this view geometrical considerations are sufficient to determine the radial dependence of the fluctuations, which influence the large scale dynamics only through perturbative wave-pressure effects and slow laminar dissipation. This perspective has often been motivated [Dobrowolny et. al, 1980a] by the occurrence of periods of highly Alfvenic fluctuations such as those reported by Belcher and Davis [1971]. It has been suggested that the linearization of incomressible MHD which obtains for exactly correlated velocity and magnetic fields allows large amplitude fluctuations to propagate outward without wave-wave interactions [Dobrowolny et al., 1980b]. One would then need to understand why the solar wind is generated with waves propagating in the outward direction only, and plausible scenarios for this have been proposed. However, there are a number of difficulties with this model, which because it lacks spectral transfer, is not turbulent at all. However, this lack of turbulence would be consistent with the view that nonlinear interactions occur in the solar corona only below the critical point. Thus only outward propagating waves, with their remnant turbulence frozen in, actually escape into the interplanetary medium. This 
picture is not ruled out by any of our analyses, except perhaps the observation that there are times when the cross helicity spectrum suggests the presence of both inward and outward directed fluctuations in the inertial range.

In the previous section we have noted that even highly Alfvenic periods sometimes show admixtures of fluctations correlated in the sense opposite to that of outward propagation. In terms of rugged invariants, some wavelengths have the opposite sign of cross helicity. These modes reintroduce nonlinearities in the dynamics and at this time we do not know if their levels are high enough to reinstate turbulent cascade processes. Recently, however, we have reported [Matthaeus, Goldstein and Montgomery, 1982] preliminary two dimensional MHD results which suggest that levels of cross helicity even higher than those typically seen in Alfvenic periods are inadequate to prevent the development of a power law inertial range. In fact, the simulations show that the resulting nonlinear processes "almost always" act to enhance the initial alignment of magnetic and velocity fields. Even if coronal dynamics preferentially generate fluctuations correlated in the outward propagating sense, it is likely that turbulence proceeds.

A second perspective is that fluctuations are initially produced near the sun and subsequently participate in turbulent decay processes superposed on the overall heliospheric expansion, but do not couple in any direct way to the mean solar wind fields. In this case interplanetary turbulence can be studied somewhat independently of other solar wind processes.

A third possiblity, which we suggest may be the most realistic, is that the initially decaying turbulence is "stirred" or forced by interactions of the local fluctuations with mean field gradients and organized structures such as high speed streams and magnetic clouds. This has been previously considered, for example by coleman [1968]. Turbulence of this type is an intrinsic part of the overall heliospheric dynamical system and quantities such as ohmic heating rates and the radial dependence of the mean magnetic field cannot be adequately accounted for without incorporating the turbulence at some level. By way of analogy, hydrodynamicists have traditionally recognized that high Reynolds number shear flows can be properly understood only when turbulence effects are included [see e.g., Tritton, 1977]. While even the existence of an effective coupling mechanism to pump turbulence at the expense of energy loss in the mean fields must be viewed as conjectural at this time, interaction regions at the leading edge of high speed streams are one class of candidates for the location where this occurs. Other possible "stirring" mechanisms include solar transients and magnetic clouds. In interaction regions, strong gradients in flow speed may stretch field lines, producing dynamo action as well as giving rise to Kelvin-Helmhotz instabilites. The typically large variance of the fluctuations in interaction regions relative to average conditions also draws our attention to them as localized sources of turbulence.

Any assessment of the role of turbulence processes in the solar wind must include a determination of the time scales over which the turbulence occurs. This is well beyond our reach at this time since we cannot measure or predict forcing, dissipation or energy transfer rates without introducing unmotivated assumptions. However, a nonlinear time can be constructed by forming the ratio of the scale size of the energy containing structures to their characteristic speed. In hydrodynamics this 'eddy turnover time' is an estimate of the lifetime of the system and the rate of relaxation of the excitations through cascade to smaller scales and subsequent dissipation. Using a scale of $10^{11}$ $\mathrm{cm}$ and a fluctuation speed of $30 \mathrm{~km} / \mathrm{sec}$ gives an estimate of the MHD eddy 
turnover time of $3 \times 10^{4} \mathrm{sec}$, appropriate to nominal conditions at 1 AU. Comparing this with the transit time of $350 \mathrm{~km} / \mathrm{sec}$ solar wind over an A.U. indicates that ten eddy turnover times are incurred for each AU of outward solar wind convection. This suggests that turbulence processes can be observed by $1 \mathrm{AU}$ and that fully developed turbulence should be observed in transit to $10 \mathrm{AU}$.

The fact that observed spectra of rugged invariants are qualitatively reproducible does not allow us to unambiguously conclude that turbulence is active in the solar wind. It is possible to argue that the observed power law spectra are due to noninteracting Alfven waves. The presence of MHD structures over a wide range of spatial scales, while often associated with turbulence, is not a sensitive enough indicator to distinguish actively evolving turbulence from noninteracting waves. Continual spectral transfer from large to small scales is an essentially turbulent feature, but it is one which analysis techniques developed so far have been unable to evaluate.

Every aspect of our understanding of solar wind turbulence is primitive enough at this stage that it is fair to say that theory and observation will both have to evolve considerably before the subject is judged as complete. Problems associated with widely varying spatial scales need to be addressed. Inclusion of turbulence modelling in the large scale heliospheric expansion equations [Hundhausen, 1972; Holzer, 1979] may contribute to the resolution of heating and radial dependence problems. Systematic radial dependences of large scale fluctuations such as the apparent coalescence of stream structure [Burlaga, these proceedings] may be related to inverse cascade or selective decay processes. At the very smallest scales the dissipation mechanism and dissipation spectra are not well understood. We anticipate that the resolution of some of these issues will play a role in our future understanding of the solar wind plasma and perhaps turbulent plasmas in general.

This research was supported, in part by the NRC/NAS Research Associateship Program, the NASA Solar Terrestrial Theory Program, NASA Grant NSG-7416 and the ISEE Guest Investigator Program.

\section{References}

Batchelor, G. K., Theory of Homogeneous Turbulence, Cambridge Univ. Press, 1970.

Belcher, J. W. and L. Davis, Large amplitude Alfven waves in the interplanetary medium, 2, J. Geophys. Res., 76, 3534, 1971.

Coleman, P. J., Wave-like phenomena in the interplanetary medium, Pl. Sp. Sci., 15, 953, 1967.

Coleman, P. J., Turbulence, viscosity and dissipation in the solar wind plasma, Astrophys. J., 153, 371, 1968.

Dobrowolny, M., A. Mangeney and P. Veltri. Properties of magnetohydrodynamic turbulence in the solar wind, Astron. Astrophys., 83, 26, 1980a.

Dobrowolny, M., A. Mangeney and P. Veltri, Fully developed anisotropic hydromagnetic turbulence in interplanetary space, Phys. Rev. Letters. 45 . $144,1980 \mathrm{~b}$.

Frisch, U., A. Pouquet, J. Léorat and A. Mazure, Possibility of an inverse cascade of magnetic helicity in magnetohydrodynamic turbulence, J. Fluid Mech., 68, 769, 1975.

Fyfe, D., and D. Montgomery, High beta turbulence in two dimensional magnetohydrodynamics, J. Plasma Phys.., 16, 181, 1976.

Grappin, R., U. Frisch, J. Léorat and A. Pouquet, Alfvenic fluctuations as asymptotic states of MHD turbulence, Astron. Astrophys., 105, 6, 1982. 
Holzer, T. E., The solar wind and related astrophysical phenomena, in Solar System Plasma Physics, ed. C. F. Kennel, L. J. Lanzerotti and E. N. Parker, vol. 1, North-Holland, 1979.

Hoppe, M. M., C. T. Russell, L. A. Frank, T. A. Eastman, and E. W. Greenstadt, Upstream hydromagnetic waves and their association with backstreaming ion populations: ISEE 1 and 2 observations, J. Geophys. Res., 86, 4471, 1981.

Hundhausen, A. J., Coronal Expansion and Solar Wind, Springer-Verlag, 1972.

Matthaeus, W. H., and D. C. Montgomery, Selective decay hypothesis at high mechanical and magnetic Reynolds numbers, Ann. N. Y. Acad. Sci., 357, 203. 1980.

Matthaeus, W. H., and M. L. Goldstein, Stationarity of magnetohydrodynamic fluctuations in the solar wind, J. Geophys. Res., 87, 10347, 1982a.

Matthaeus, W. H., and M. L. Goldstein, Measurement of the rugged invariants of magnetohydrodynamic turbulence in the solar wind, J.Geophys. Res., 87, 6011, 1982b.

Matthaeus, W. H., M. L. Goldstein and D. C. Montgomery, Dynamic alignment of velocity and magnetic fields in magnetohydrodynamic turbulence, E0S, 63. 1069, 1982.

Panchev, S., Random Functions and Turbulence, Pergammon Press, 1971.

Sari, J. W. and N. F. Ness, Power spectra of the interplanetary magnetic field, Solar Phys., 8, 155, 1969.

Smith, C. W., M. L. Goldstein, and W. H. Matthaeus, Turbulence analysis of the Jovian upstream wave phenomenon, J. Geophys. Res., in press, 1983.

Tritton, D. J., Physical Fluid Dynamics, Van Nostrand Reinhold, 1977. 